\title{
Diagnosis and Treatment of Demodecosis with Secondary Bacterial Infection in a Pug-A Case Presentation
}

\author{
G. Ambica ${ }^{1}$, G. Abhinav Kumar Reddy ${ }^{2}$, Vinay M. Ratnalikar ${ }^{3}$, Sandepogu Ranjith \\ Kumar $^{4}$, Keshamoni Ramesh ${ }^{5}$ and Maramulla Aruna ${ }^{6}$
}

Department of Veterinary Medicine, College of Veterinary Science, Rajendranagar, Hyderabad-500030, Telangana state, India

*Corresponding author

\section{A B S T R A C T}

\section{Ke y w o r d s \\ Pug, Demodicosis, \\ Pyoderma, \\ Alopecia, \\ Treatment.}

\section{Article Info}

Accepted:

15 April 2020

Available Online:

10 May 2020
Skin infections are most common in dogs, especially pugs are more prone due to its folded skin nature. A Pug of 3 years age weighing around $10 \mathrm{kgwas}$ brought to the Veterinary Clinical Complex, Rajendranagar, Hyderabad with the complaint of alopecia, pruritus, decrease in the appetite and erythematous lesions all over the body. Detailed clinical examination revealed mild increase in temperature $\left(102.5^{\circ} \mathrm{F}\right)$ and normal heart rate, pulse and respirations. Skin scrapings and bacterial cultural examination revealed mixed infection of Demodicosis and Staphylococcal pyoderma. Hence, both antiparasitic and antibacterial medication was started in a proper way along with anti-inflammatory and anti-allergic agents and also supportive therapy with Omega 3, Omega 6 fatty acid supplements, immune boosters and hypoallergenic diet. Animal showed improvement from next day of the therapy and treatment was continued with some modifications for two months. Dog recovered completely and was confirmed by the negative findings in both skin scrapings and bacteriological culture examination complete alleviation of skin lesions.

\section{Introduction}

Canine demodicosis is a refractory dermatopathic consequence in the dog as results of the pathological proliferation of Demodex mites predominantly present in the hair follicles. Canine demodicosis (Follicular Mange) is an inflammatory skin disorder in dogs associated with higher than normal populations of Demodectic mites (Mueller, 2014). The Demodex mite is skin commensal transmitted from bitch to puppies in the first few days after birth (Gortel, 2006). It has a very harmful effect on the health, utility and cosmetic values in dogs (Chatterjee, 2007). Clinical disease is influenced by numerous such as genetic defect, alteration of skin's structure, immunological disorders, hormonal status, breed, age, nutritional status, oxidative stress, endoparasites and debilitating diseases but the immune status is thought to be the most significant factor among all (Shanker 
Singh \& Umesh Dimri, 2014). Immunosuppression allows the mites to proliferate excessively in hair follicles, resulting in clinical signs (Greve \& Gaafar, 1966). The major clinical signs include mild erythema, itching, partial or complete alopecia, comedones and Pruritis (Ralf, et al., 2011-12). The disease is mostly caused by mite Demodex canis, however others mites species like Demodex injai and Demodex cornei may also cause the disease (Tater \& Patterson, 2008).

The disease occurs in nature in two forms: localized and generalized (Scott, et al., 2001). A generalized form is usually associated with secondary bacterial infection and requires vigorous and prolonged treatment (Mueller, 2012). Diagnosis is rapidly and reliably confirmed by finding more than one mites per microscopic field in deep skin scraping (Soulsby, 1968). Treatment in such cases should target both mite and bacteria. Appropriate oral antibiotic therapy and contemporaneous topical antimicrobial therapy (whole-body soaks or shampoos) is indicated in generalized demodicosis with secondary bacterial infection along with topical application of Benzoyl peroxide and chlorhexidine-based shampoos (Kwochka KW \& Kowalski, 1991). Topical therapy with shampoo removes crusts and debris that may contain mites, exudate and inflammatory mediators thus reduces the recovery time.

\section{Materials and Methods}

A three years Pug weighing around $10 \mathrm{~kg}$ was brought to the Veterinary Clinical Complex, Rajendranagar with the complaint of alopecia, pruritus, decrease in the appetite and erythematous lesions all over the body for one month. Clinical examination revealed mild increase in temperature $\left(102.5^{\circ} \mathrm{F}\right)$, normal heart rate, pulse and respirations. Detailed examination of skin revealed erythema, pyoderma, alopecia and erosions and lesions on face, around the eyes and ears, chin region, fore limbs, hind limbs neck and lateral and ventral abdomen [Fig.1,2 and3].

Skin scrapings were collected with scalpel blade and collection of scrapings was continued until there was slight ooze of blood from dermal capillaries. Skin scrapings were taken in $10 \% \mathrm{KOH}$ and were heated gently till hair gets digested and then centrifuged @ $3000 \mathrm{rpm}$ for five minute. The sediment was examined under low and high power (10X and 40X) of microscope. Skin surface impressions collected were using sterile swab for cultural examination.

\section{Results and Discussion}

Microscopic examination of the skin scrapping was found positive for the skin mite Demodex canis (Fig 4). Cultural examination of skin surface impressions on MSA revealed presence of clusters of Gram positive cocci and were confirmed by Grams staining [Fig.5 and 6].

A multimodal approach was undertaken for the therapy with both antiparasitic and antibacterial medication in a proper way along with anti-inflammatory and anti-allergic agents and also supportive therapy. Dog was treated with oral Tab. Neomec (Ivermectin) $10 \mathrm{mg} @ 600 \mu \mathrm{g} / \mathrm{kg}$ initially for 10 days and

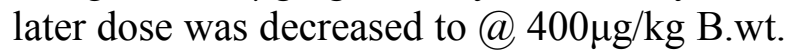
and was continued for one and half month. Tab. Cephalixen@20mg/kg B.wt. BID was given to control pyoderma resulted due to secondary bacterial infection. Advised bath with Sulbenz pet shampoo once in a week which contains Bezoyl Peroxide - 2.5\% w/vused to remove crust and debris from the skin; keratolytic agents, Micronized Sulphur $2 \%$ w/v and Salicylic Acid - 1\% w/v which causes the skin to shed dead cells from its top layer by increasing the amount of moisture in 
the skin and dissolving the substance that makes the cells clump together. This effect makes it easier to shed the skin cells, softens the top layer of skin, and decreases scaling and dryness and Zinc oxide acting as soothing agents. Prescribed Vet-pro Hypoallergenic diet BID which contains optimum levels of omega 3 and 6 fatty acids for healthy skin and coat along with the added organic zinc and selenium, aloevera extract and bromelain for natural skin defense and to promote healing.

Topical application of Charmil ointment containing Allium sativum, Azadiractaindica, twice day and it has got an antibacterial andanti-inflammatory action against inflamed skin along with antipruritic action for alleviating itching and irritation.Pup also given Nutricoat syrup twice daily, containing essential fatty acids, Linoleic (Omega 6) and Linolenic (Omega 3) acids to minimize oxidative stress along with the other vital nutrients like biotin, Zinc, selenium, vitamins A, D3 and E to supplement the action of essential fatty acids in maintaining the skin and coat condition.

Other supportive therapy with Immune booster liquid, Immunol given@3ml p/o thrice daily and antihistaminic tablet, Levocetrizine @ $10 \mathrm{mg}$ once daily for alleviating intense pruritus. Dog started showing the response from next day of the therapy and treatment was continued for a period of 2 months. Dog recovered completely and was confirmed by the negative findings in both skin scrapings and bacteriological culture examination complete alleviation of skin lesions (Fig.8 and 9).

Demodicosis, also known as red mange, is an infestation of the dog by a variety of demodex mite. Demodex mites are considered normal residents of canine skin and have long been recognized as the most common species in dogs. Cutaneous changes in young and older patients include comedones, papules, pustules, follicular casts, plaques, crusts, edema, and deep folliculitis and furunculosis (Mueller, 2004). It resides in the hair follicles and sebaceous glands and survives on epidermal debris, cells and sebum (Miller WH et al., 1993).

An impaired immune system causes the proliferation of the mite leading to infestation. A multiplying mite secretes a humoral factor which suppresses the immune response against the parasite, thus allowing its proliferation (Ginel P. Demodicosebeim Hund, 1996). In the current case, the clinical manifestations like alopecia with follicular pustules, moist and hemorrhagic exudation were observed on the entire face and forelimbs around ears and eyes and in the interdigital space, pustules with draining tract were in agreement with the earlier authors (Sarkar P et al., 2004, Ballari S et al., ) $)^{16,}$ ${ }^{15}$.Most cases involve a secondary bacterial skin infection, which needs administration of systemic antibiotics for several weeks concomitantly with the acaricidal treatment (Verde Maite, 2005).

When demodicosis is complicated by bacterial infection, therapeutic regime requires a strategic approach that overcomes the effects of both mite and bacteria to the animal. Proper timing, frequency and duration of treatment will determine the outcome of therapy (Yatoo, et al., 2014). The essential fatty acids are useful for maintaining the health of the coat and help the pet to fight against allergies and various skin problems. Demodicosis is associated with oxidative stress that predisposes dog for rapid proliferation of mites; hence in current case supportive therapy with essential fatty acids was given.

Further, Ivermectin given for the current case is with macrocyclic lactone which kills the 
parasite by potentiating gamma-aminobutyric acid (GABA)-gated chloride channels resulting in increased permeability (Fondati, 1996). Ivermectin is usually dosed at 300 to $600 \mathrm{lg} / \mathrm{kg} / \mathrm{d}$ orally for treatment of generalized canine demodicosis (Ristic, et al., 1995). The shampoo Sulbenzpet, recommended for the present case containing Benzoyl Peroxide is a good keratolytic agent and has strong follicular flushing action which assists in clearance of mites from skin of dogs (Satish Kumar et al., 2017).
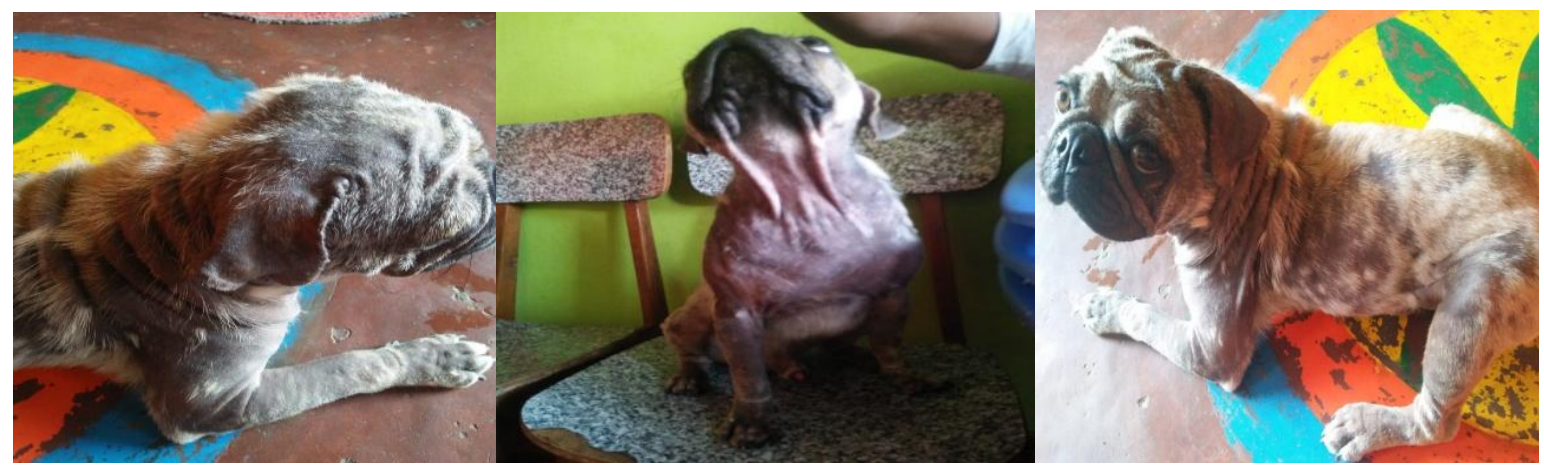

Fig.1,2\&3 Erythema and alopecia on face, around the eyes and ears; Chin region, fore limbs, neck and ventral abdomen; Hind limbs and lateral abdominal areas

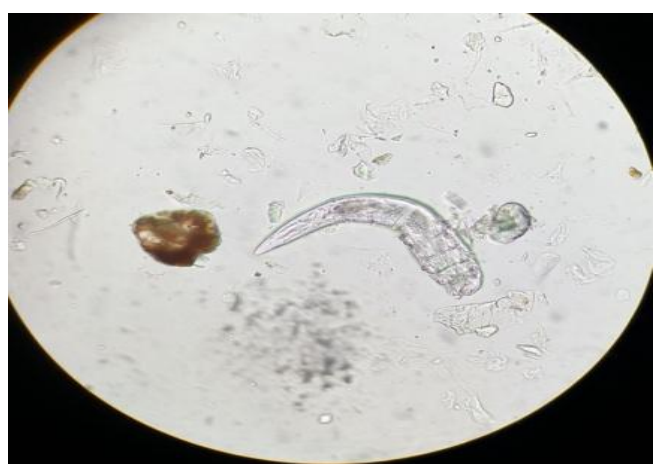

Figure.4 Demodex canis in skin scrapings under microscope

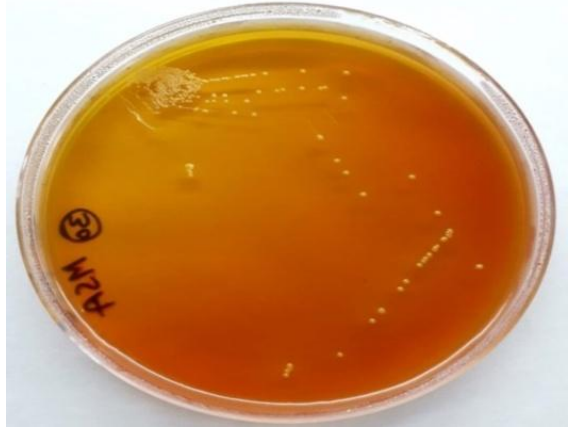

Figure.5 Staphylococcus showing golden pigmentation on MSA

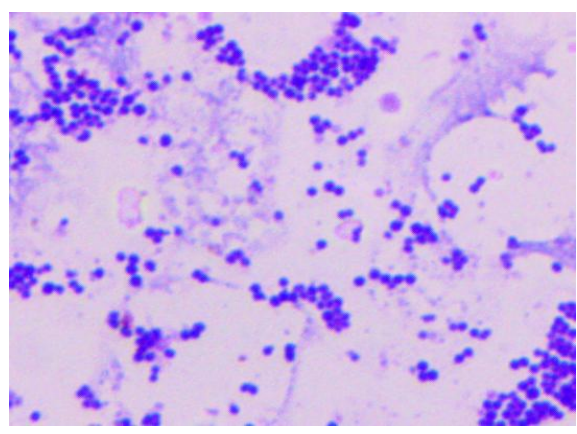

Figure.6 Microscopic view of staphylococcus by Grams staining 


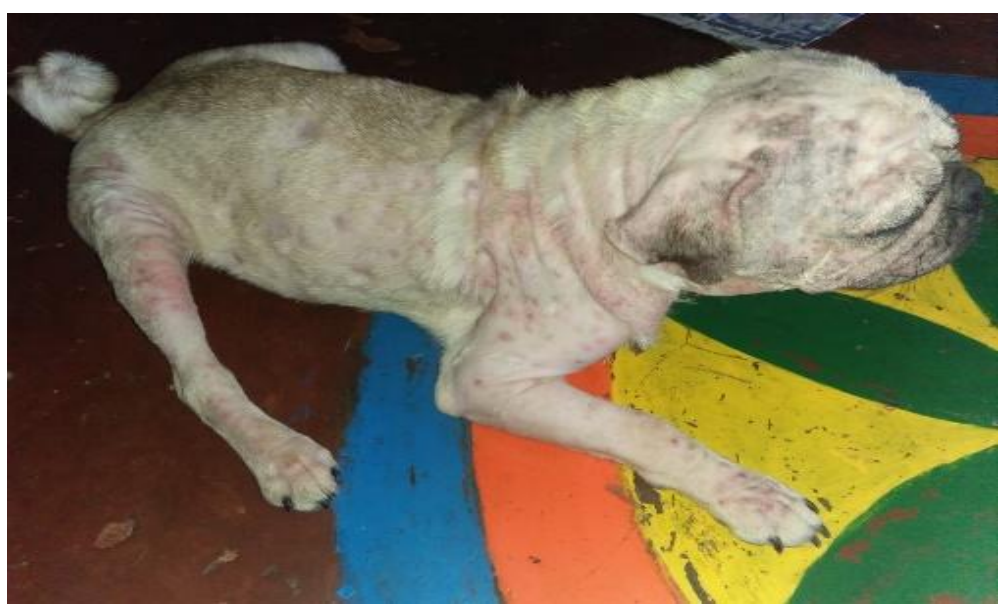

Figure.7 After one and half month of therapy

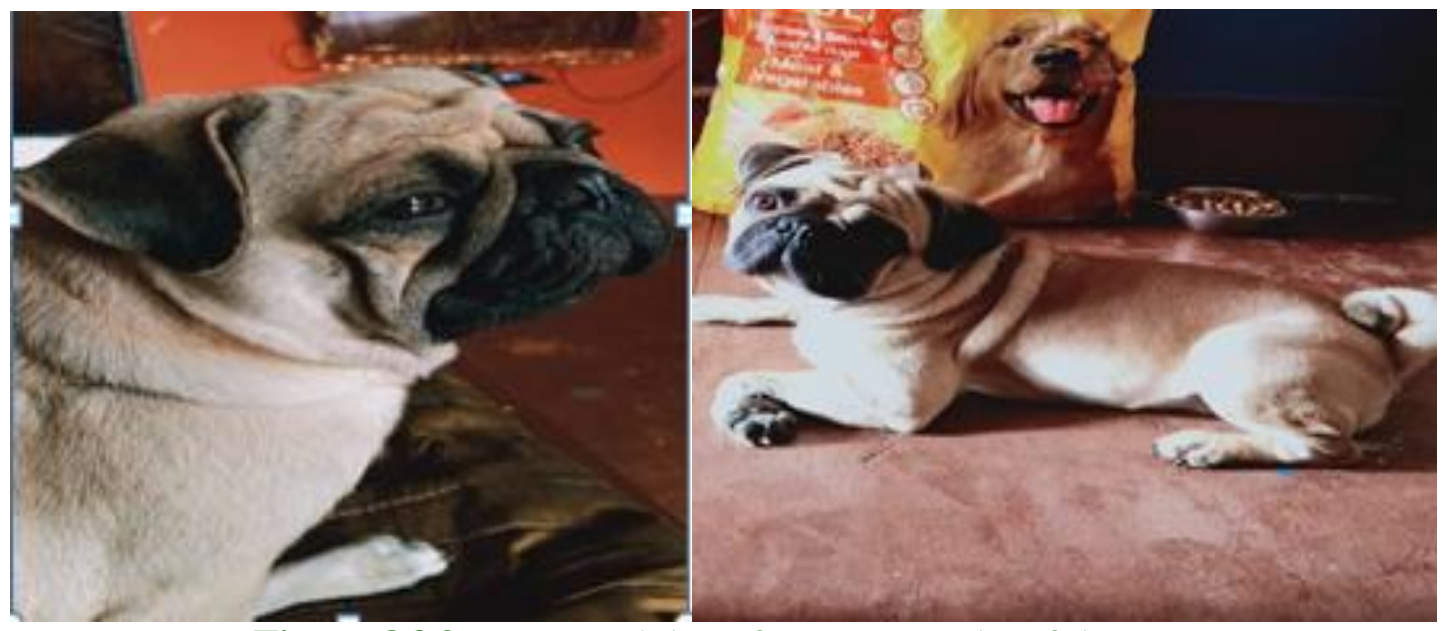

Figure.8\&9 Recovered dog after two months of therapy

With the present case, it can be concluded that the cases of demodicosis if not treated in time can become complicated with secondary bacteria leading to pyoderma and such cases can effectively managed with administration of ivermectin, and antibiotics along with supportive therapy with antihistamines, anti-inflammatory drugs, hypoallergenic prescription diet and bathing with benzoyl peroxide containing shampoo.

\section{Acknowledgement}

The author wish to express sincere thanks to Dr K. Satish Kumar, Professor \&University Head, Department of Veterinary Medicine, College of Veterinary Science, PVNR TVU and other staff for providing the facilities and assisting successful completion of this case study.

\section{References}

Ballari S, Balachandran C, George VT, Manohar BM. Pathology of canine demodicosis.

Chatterjee S. Studies on the incidences and treatment of demodectic mange infection in dogs with special reference to its herbal therapy. M.V.Sc. Thesis submitted to W.B.U.A.F.S., Kolkata, West Bengal, 2007.

Fondati A. Efficacy of daily oral ivermectin in the treatment of 10 cases of generalized demodicosis in adult dogs. Veterinary Dermatology. 1996; 7:99-104. 
Ginel P. Demodicosebeim Hund. [Demodicosis in dogs] [in German]. Waltham Focus. 1996; 6: 2-7.

Gortel K. Update on canine demodicosis. Veterinary Clinics: Small Animal Practice. 2006; 36(1):229-41.

Greve JH, Gaafar SM. Natural transmission of Demodex canis in dogs. Journal of American Veterinary Medical Association. 1966; 148:1043-1045.

Kwochka KW, Kowalski JJ. Prophylactic efficacy of four antibacterial shampoos against Staphylococcus intermedius in dogs. American Journal of Veterinary Research. 1991; 52:115-118.

Miller WH, Jr, Scott DW, Wellington JR, Panic R. Clinical efficacy of milbemycin oxime in the treatment of generalized demodicosis in adult dogs. Journal of the American veterinary medical association. 1993; 203:1426- 1429.

Mueller RS, Vet Dermatol. Edn. 2014; 15(2):7589.

Mueller RS. Applied dermatology: an update on the therapy of canine demodicosis. Compendium, 2012, 34(4).

Mueller RS. Treatment protocols for demodicosis: An evidence-based review. Vet Dermatol 2004; 15:75-89.

Ralf S, Mueller EB, Lluís F, Birgit H, Stephen L, Manon $\mathrm{P}$ et al., Shipstone. Treatment of demodicosis in dogs: clinical practice guidelines. Veterinary Dermatology. 2011-2012; 23(2):86.

Ristic Z, Medleau L, Paradis M, white-Weithers NE. Ivermectin for the treatment of generalized demodicosis in dogs. Journal of the American Animal Hospital Association. 1995; 207:1308-10.

Sarkar P, Mukherjee J, Ghosh A, Bhattacharjee
M, Mahato S, Chakraborty A, Mondal M, Banerjee C, Chaudhuri S. A comparative analysis of immuno restoration and recovery with conventional and immunotherapeutic protocols in canine generalized demodicosis: a newer insight of immunotherapeutic efficacy of T11TS. Immunological investigations. 2004; 33(4):453-68

Satish Kumar, Mrigakshi Yadav, Vidhi Kunwar, Penny Arya, Radhika Prakash Bhatt, Upadhyaya AK. Therepeutic managmemt of mycotic demodecosis in a dog-a case report. Indian Journal of Canine Practice. 2017; 9(2):117-118.

Scott DW, Miller WM, Griffin CE. Parasitic skin diseases. In: Muller, K. (ed) Small animal dermatology, 6th ed. W.B. Saunders, Philadelphia, 2001, 423-516.

Shanker K Singh, Umesh Dimri. The immunopathological conversions of canine demodicosis. Veterinary Parasitology. 2014; 203(1-2):1-5.

Soulsby EJL. Helminths, arthropods and protozoa of domesticated animals (of MSnnig's Veterinary helminthology and entomology). Helminths, arthropods and protozoa of domesticated animals (sixth edition of MSnnig's Veterinary helminthology and entomology), 1968.

Tater KC, Patterson AC. Canine and feline demodicosis. Vet. Med, 2008, 444-461.

Verde Maite, Canine Demodicosis. Treatment Protocol. The North American Veterinary Conference - Proceedings. 2005; 287:3.

Yatoo MI, Jhambh R, Malepad DP, Kumar P, Dimri U. Advances in therapeutic management of complicated demodicosis in canines. J Adv. Parasitology. 2014; 1(1):6-8./

\section{How to cite this article:}

Ambica. G., G. Abhinav Kumar Reddy, Vinay M. Ratnalikar, Sandepogu Ranjith Kumar, Keshamoni Ramesh and Maramulla Aruna. 2020. Diagnosis and Treatment of Demodecosis with Secondary Bacterial Infection in a Pug-A Case Presentation. Int.J.Curr.Microbiol.App.Sci. 9(05): 1753-1758. doi: https://doi.org/10.20546/ijcmas.2020.905.197 